\title{
Calculating disability-adjusted-life-years lost (DALYs) in discrete-time
}

Bruce A Larson ${ }^{1,2}$

\begin{abstract}
Disability-adjusted-life-years lost (DALYS) is a common outcome metric for cost-effectiveness analyses, and the equations used for such calculations have been presented previously by Fox-Rushby and Hanson (see, e.g., "Health Policy and Planning 16:326-331, 2001"). While the equations are clear, the logic behind them is opaque at best for a large share of public health practitioners and students. The objective of this paper is to show how to calculate DALYs using a discrete time formulation that is easy to teach to students and public health practitioners, is easy to apply for those with basic discounting skills, and is consistent with the discounting methods typically included on the costing side of cost-effectiveness analysis. A continuous-time adjustment factor is derived that can be used to ensure exact consistency between the continuous and discrete time approaches, but this level of precision is typically unnecessary for cost-effectiveness analyses. To illustrate the approach, both a new, simple example and the same example presented in Fox-Rushby and Hanson are used throughout the paper.
\end{abstract}

Keywords: Disability adjusted life years lost, Discrete time, Cost-effectiveness

\section{Background}

The concept of disability adjusted life years lost (DALYs) was introduced in the 1993 World Development Report as a way to estimate and compare the burden of morbidity and premature mortality caused by widely varying conditions and states within and among countries [1]. DALYs are calculated as the present discounted value of future years of healthy life lost to morbidity/disability and future years of life lost to premature mortality $[2,3]$. Building on this earlier literature, Fox-Rushby and Hanson, hereafter referred to as FRH, provided a clear standard for calculating DALYs as an outcome indicator for cost-effectiveness analyses [4]. FRH provide the rather arduous equations for years of life lost (YLL) and years lived disabled (YLD), which are an artifact of the continuous-time math used in the development of DALYs. Over the past twenty years, a wide range of analyses in developed and developing countries have used DALYs in cost-effectiveness analyses [5-13].

Calculating the present discounted value of a stream of future costs or benefits is a standard topic in most

\section{Correspondence: blarson@bu.edu}

${ }^{1}$ Department of International Health, Boston University School of Public Health, 801 Massachusetts Avenue, Boston, MA 02118, USA

${ }^{2}$ Center for Global Health and Development, Boston University, 801

Massachusetts Avenue, Boston, MA 02118, USA courses that include economic evaluation and costeffectiveness. Time is typically measured in discrete units (months, years, etc.), and present values are calculated easily in a spreadsheet program. Discrete time units are also typically the denominator in reported economic and health data (e.g. annual program costs, annual mortality figures, and so on). While useful in most other ways, however, discrete time units are not consistent with the traditional calculation of DALYs with time as a continuous variable [14].

The purpose of this paper is to present a very simple approach for calculating DALYs in discrete time for cost-effectiveness analyses. Calculating DALYs using a discrete time formulation is easy to implement in a spreadsheet program and provides sufficient precision for most users' purposes. This approach is easy to teach to students and public health practitioners, is easy to apply for those with basic discounting skills, is consistent with the discounting methods typically included in the costing side of cost-effectiveness analysis, and all the assumptions imbedded in the analysis are very transparent. The approach is applied to a new simple example as well as to the same worked-out example provided in FRH [4]. 


\section{The discrete-time approach}

The costing component of cost-effectiveness analyses typically uses standard, discrete-time discounting in the calculation of the present value of program costs and/or the annual equivalent costs for multi-year programs [15]. With time in years, the discrete time discount factor, $1 /(1+r)^{t}$, where $r$ is the discount rate and the number of years, is commonly used.

The following simple example will be used throughout the paper. Suppose a man dies on his $55^{\text {th }}$ birthday, and age-specific additional life expectancy for this age was 10 years in his country. Without discounting, 10 years of life are lost, so $\operatorname{YLL}(0,0)=10$.

With 10 years of life lost and a discount rate of $3 \%$, Table 1 shows how a spreadsheet is typically organized to calculate the present discounted value of a stream of numbers (e.g., costs, payments, or in this case years of life lost). In Table 1, the 'present' is time 0 , meaning the time at which the evaluation is being completed. Having time progress from 0 to 9 assumes that each of the 10 years lost is counted at the beginning of the year, which is the same as stream of payments where each payment is made at the beginning of the year. Using this calculation for the 10 years of life lost, $\operatorname{YLL}(3,0)=$ 8.786 with discrete time discounting.

How does this answer compare to the continuoustime approach? In the absence of age weighting, the equation for years of life lost due to premature mortality provided in FRH reduces to:

$$
Y L L(r, 0)=\int_{0}^{L} 1 * e^{-r t} d t=\left(\frac{1}{r}\right)\left(1-e^{-r L}\right)
$$

where 0 is the 'present', $\mathrm{L}$ is age-specific life expectancy for the person who died prematurely, representing the years of

Table 1 Calculating $\operatorname{YLL}(3,0)$ for a person who dies on their $55^{\text {th }}$ birthday with age-specific life expectancy of 10 years with annual discounting as $1 /(1+r)^{t}$

\begin{tabular}{ccccc}
\hline $\begin{array}{c}\text { Which year } \\
\text { was lost }\end{array}$ & $\mathbf{t}$ & $\begin{array}{c}\text { Year } \\
\text { lost }\end{array}$ & $\begin{array}{c}\text { Discrete time } \\
\text { discount factor }\end{array}$ & $\begin{array}{c}\text { Discounted } \\
\text { year lost }\end{array}$ \\
\hline 56 & 0 & 1 & $\mathrm{~B}=1 /(1+0.03)^{\mathrm{t}}$ & $\mathrm{C}=\mathrm{A}^{*} \mathrm{~B}$ \\
57 & 1 & 1 & 1.000 & 1.000 \\
58 & 2 & 1 & 0.971 & 0.971 \\
59 & 3 & 1 & 0.943 & 0.943 \\
60 & 4 & 1 & 0.915 & 0.915 \\
61 & 5 & 1 & 0.888 & 0.888 \\
62 & 6 & 1 & 0.863 & 0.863 \\
63 & 7 & 1 & 0.837 & 0.837 \\
64 & 8 & 1 & 0.813 & 0.813 \\
65 & 9 & 1 & 0.789 & 0.789 \\
& Sum of & 10 & Sum of discounted & 8.786 \\
& years lost & & year lost column & \\
\hline
\end{tabular}

life lost for the person who died, and $\mathrm{r}$ is the discount rate. For notational simplicity $\operatorname{YLL}(r, 0)$ is used for years of life lost in Equation (1). With a 3\% discount rate and 10 years of life expectancy, the answer to Equation (1) is 8.6394.

The basic point here is that the $1.7 \%$ difference between a simple discrete-time approach and the continuous time approach is minor, and such differences will not change fundamentally any ranking in a cost-effectiveness analysis. In addition, anyone who has actually completed the costing component of a cost-effectiveness analysis knows how many assumptions, estimates, and outright guesses are included in the cost analysis. The age-specific life expectancy figures inherent in DALY calculations similarly rely on data of varying quality, and the same kinds of uncertainties affect estimates of the change in DALYs due to an intervention or policy change as well.

\section{The FRH DALY $(3,0)$ example using this simple approach}

Another benefit of the simple approach is that a more complicated scenario can easily be incorporated into the same spreadsheet, no equations needed. FRH consider a woman who develops bipolar depression at age 35, lives for 10 years with the disorder, and then dies prematurely at age 45 . The disability weight is 0.60 , life expectancy at age 45 is 34.73 years (age 79.73), and the "present" for the present value calculation is age 35 (meaning exactly on her $35^{\text {th }}$ birthday).

Table 2 shows the spreadsheet used to calculate DALY $(3,0)$ for this example without age weighting (see the appendix for age weighting). From time 0 through time 9 , representing her $36^{\text {th }}-45^{\text {th }}$ years of life, she loses 0.6 years of life annually. From time 10 through 43, representing her $46^{\text {th }}-79^{\text {th }}$ years of life, she loses 1 year of life annually. In time $44\left(80^{\text {th }}\right.$ year of life), she loses 0.73 years of life. Using the discrete time discount factor $1 /(1+r)^{t}$, the present value of the stream of lost years of life $(0.60$ per year when disabled plus 1.00 per year when deceased) is 21.666 .

How does the answer from this simple approach compare to the FRH answer? For the 10 years lived disabled, we have:

$$
Y L D(3,0)=0.6\left(\frac{1}{0.03}\right)\left(1-e^{-0.03 * 10}\right)=5.1836
$$

For the 34.73 of life lost, we have:

$$
Y L L(3,0)=e^{-0.03 * 10}\left(\frac{1}{0.03}\right)\left(1-e^{-0.03 * 34.73}\right)=15.9823
$$

Combining the answers in Equation (2) and (3), DALY $(3,0)$ using the continuous time approach is 21.166. Thus, 
Table 2 Calculating $\operatorname{DALY}(3,0)$ for a women who develops bipolar disorder on 35th birthday, lives 10 years, and life-expectancy at age 45 is $\mathbf{3 4 . 7 3}$ years, with annual discounting as $1 /(1+r)^{t}$

\begin{tabular}{|c|c|c|c|c|}
\hline $\begin{array}{l}\text { Which } \\
\text { year } \\
\text { was lost }\end{array}$ & $t$ & $\begin{array}{l}\text { Year lost (either } \\
\text { disability weight } \\
\text { or death) }\end{array}$ & $\begin{array}{l}\text { Discrete time } \\
\text { discount } \\
\text { factor }\end{array}$ & $\begin{array}{c}\text { Discounted } \\
\text { year lost }\end{array}$ \\
\hline 36 & 0 & 0.6 & 1.000 & 0.600 \\
\hline 37 & 1 & 0.6 & 0.971 & 0.583 \\
\hline 38 & 2 & 0.6 & 0.943 & 0.566 \\
\hline 39 & 3 & 0.6 & 0.915 & 0.549 \\
\hline 40 & 4 & 0.6 & 0.888 & 0.533 \\
\hline 41 & 5 & 0.6 & 0.863 & 0.518 \\
\hline 42 & 6 & 0.6 & 0.837 & 0.502 \\
\hline 43 & 7 & 0.6 & 0.813 & 0.488 \\
\hline 44 & 8 & 0.6 & 0.789 & 0.474 \\
\hline 45 & 9 & 0.6 & 0.766 & 0.460 \\
\hline 46 & 10 & 1 & 0.744 & 0.744 \\
\hline 47 & 11 & 1 & 0.722 & 0.722 \\
\hline 48 & 12 & 1 & 0.701 & 0.701 \\
\hline 49 & 13 & 1 & 0.681 & 0.681 \\
\hline 50 & 14 & 1 & 0.661 & 0.661 \\
\hline 51 & 15 & 1 & 0.642 & 0.642 \\
\hline 52 & 16 & 1 & 0.623 & 0.623 \\
\hline 53 & 17 & 1 & 0.605 & 0.605 \\
\hline 54 & 18 & 1 & 0.587 & 0.587 \\
\hline 55 & 19 & 1 & 0.570 & 0.570 \\
\hline 56 & 20 & 1 & 0.554 & 0.554 \\
\hline 57 & 21 & 1 & 0.538 & 0.538 \\
\hline 58 & 22 & 1 & 0.522 & 0.522 \\
\hline 59 & 23 & 1 & 0.507 & 0.507 \\
\hline 60 & 24 & 1 & 0.492 & 0.492 \\
\hline 61 & 25 & 1 & 0.478 & 0.478 \\
\hline 62 & 26 & 1 & 0.464 & 0.464 \\
\hline 63 & 27 & 1 & 0.450 & 0.450 \\
\hline 64 & 28 & 1 & 0.437 & 0.437 \\
\hline 65 & 29 & 1 & 0.424 & 0.424 \\
\hline 66 & 30 & 1 & 0.412 & 0.412 \\
\hline 67 & 31 & 1 & 0.400 & 0.400 \\
\hline 68 & 32 & 1 & 0.388 & 0.388 \\
\hline 69 & 33 & 1 & 0.377 & 0.377 \\
\hline 70 & 34 & 1 & 0.366 & 0.366 \\
\hline 71 & 35 & 1 & 0.355 & 0.355 \\
\hline 72 & 36 & 1 & 0.345 & 0.345 \\
\hline 73 & 37 & 1 & 0.335 & 0.335 \\
\hline 74 & 38 & 1 & 0.325 & 0.325 \\
\hline 75 & 39 & 1 & 0.316 & 0.316 \\
\hline
\end{tabular}

Table 2 Calculating $\operatorname{DALY}(3,0)$ for a women who develops bipolar disorder on 35th birthday, lives 10 years, and life-expectancy at age 45 is 34.73 years, with annual discounting as $1 /(1+r)^{t}$ (Continued)

\begin{tabular}{ccccc}
\hline 76 & 40 & 1 & 0.307 & 0.307 \\
77 & 41 & 1 & 0.298 & 0.298 \\
78 & 42 & 1 & 0.289 & 0.289 \\
79 & 43 & 1 & 0.281 & 0.281 \\
80 & 44 & 0.73 & 0.272 & 0.199 \\
& $\begin{array}{c}\text { Sum of } \\
\text { years } \\
\text { lost }\end{array}$ & 40.73 & $\begin{array}{c}\text { Sum of } \\
\text { discounted } \\
\text { year lost } \\
\text { column }\end{array}$ & 21.666 \\
& & & & \\
& & & &
\end{tabular}

the simple discrete time approach yields a result that is $2.4 \%$ higher than the FHR answer.

A minor adjustment for discounting in discrete time

The continuous-time approach embedded into standard DALY calculations uses $\mathrm{e}^{-\mathrm{rt}}$ as the discount factor rather than $1 /(1+r)^{t}$. In practice, $e^{-r t}$ is a little less than $1 /(1+r)^{t}$, so that present discounted values will be slightly different depending on which formulation of the discount factor is used. If $r=0.03$ is the discount rate when discounting using $\mathrm{e}^{-0.03 t}$, then a discount rate for discrete time of $\mathrm{r}^{\mathrm{d}}=\mathrm{e}^{0.03}-1=0.03045453$ will yield the identical discount factor (this point is also explained in [2]). This slightly higher discount rate, for example, reduces slightly the estimated DALYs lost in Table 2 to 21.485, which is $1.5 \%$ higher than the FRH answer.

\section{An exact adjustment for the discrete approach}

In the absence of age weighting, the simple discrete approach, perhaps using $\mathrm{e}^{-\mathrm{rt}}$ for annual discounting, is more than adequate for program evaluations and applied cost-effectiveness analyses. If, however, an analyst feels compelled to provide the 'exact' FRH answer, one simple adjustment can be made.

With the commonly assumed 3\% discount rate, one year of life is lost is:

$$
Y L L(3,0)=\left(\frac{1}{0.03}\right)\left(1-e^{-0.03}\right)=0.985148881716395
$$

In other words, one year of life lost is less than one because the year is lost continuously over the full year, instead of being counted all at once at the beginning for the year (as in Table 1 and Table 2).

Table 3 replicates the analysis in Table 1 with the addition of this continuous time adjustment factor (0.985148881716395). In Table 1 , each year of life lost is 
Table 3 Calculating $\operatorname{YLL}(3,0)$ for a person who dies on their 55th birthday with age-specific life expectancy of 10 years, annual discounting as $\mathrm{e}^{-\mathrm{rt}}$, and the continuous time adjustment factor

\begin{tabular}{cccccc}
\hline $\mathbf{t}$ & $\begin{array}{c}\text { Year } \\
\text { lost }\end{array}$ & $\begin{array}{c}\text { Continuous } \\
\text { time } \\
\text { adjustment } \\
\text { factor }\end{array}$ & $\begin{array}{c}\text { Adjusted } \\
\text { year lost } \\
\text { for } \\
\text { continuous } \\
\text { time }\end{array}$ & $\begin{array}{c}\text { Discrete } \\
\text { time } \\
\text { discount } \\
\text { factor }\end{array}$ & $\begin{array}{c}\text { Discounted } \\
\text { and } \\
\text { adjusted } \\
\text { year lost }\end{array}$ \\
\hline 0 & 1 & 0.9851 & 0.985148882 & 1.000 & 0.9851 \\
1 & 1 & 0.9851 & 0.985148882 & 0.970 & 0.9560 \\
2 & 1 & 0.9851 & 0.985148882 & 0.942 & 0.9278 \\
3 & 1 & 0.9851 & 0.985148882 & 0.914 & 0.9004 \\
4 & 1 & 0.9851 & 0.985148882 & 0.887 & 0.8737 \\
5 & 1 & 0.9851 & 0.985148882 & 0.861 & 0.8479 \\
6 & 1 & 0.9851 & 0.985148882 & 0.835 & 0.8229 \\
7 & 1 & 0.9851 & 0.985148882 & 0.811 & 0.7985 \\
8 & 1 & 0.9851 & 0.985148882 & 0.787 & 0.7749 \\
9 & 1 & 0.9851 & 0.985148882 & 0.763 & 0.7520 \\
Sum & 10 & & & Sum of & 8.6394 \\
of & & & & & discounted \\
years & & & & and adjusted & \\
lost & & & & years lost & \\
\hline & & & & & \\
\hline
\end{tabular}

multiplied by this adjustment factor, and then discounted using the $\mathrm{e}^{-\mathrm{rt}}$ formulation. With this addition to the analysis, $\operatorname{YLL}(3,0)=8.6394$, which is the exact FRH answer.

The analysis in Table 2 can also be replicated using the same approach; simply multiply the year lost or lived disabled by the continuous time adjustment factor and use $\mathrm{e}^{-\mathrm{rt}}$ for discounting. The answer is $\operatorname{DALY}(3,0)=21.165$ while the FRH approach yields 21.166. The reason for the minor discrepancy is that the final 0.73 of a year is simply weighted by the annual continuous time adjustment factor. To be exactly correct for that last 0.73 of a year, Equation (1) using $\mathrm{L}=0.73$ equals 0.7221 . If 0.7221 is used instead of 0.73 , the result becomes 21.166.

In summary, the discrete approach outlined above yields the same answer as the continuous time approach using $\mathrm{e}^{-\mathrm{rt}}$ as the discount factor and adjusting each year of death or morbidity/disability by the continuous time adjustment factor (from Equation 4).

\section{Discussion}

While disability adjusted life years have traditionally been calculated using the continuous time formulation reviewed presented in FRH, a simple approach using discrete time to calculate the present discounted value is easy to implement, easy to explain to practitioners and policy makers, and consistent with methods typically used on the costing side of cost effectiveness analysis.
Table 4 Age weights for discrete time

\begin{tabular}{|c|c|}
\hline $\mathrm{C}$ & 0.176116 \\
\hline $\bar{\beta}$ & 0.04 \\
\hline Sum of Age Weights & 100.000 \\
\hline Age (birth and future birthdays): & Age weight function: \\
\hline$a(x)$ & $a(X)=C(x+0.5) e^{-\beta(X+0.5}$ \\
\hline 0 & 0.086 \\
\hline 1 & 0.249 \\
\hline 2 & 0.398 \\
\hline 3 & 0.536 \\
\hline 4 & 0.662 \\
\hline 5 & 0.777 \\
\hline 6 & 0.883 \\
\hline 7 & 0.979 \\
\hline 8 & 1.066 \\
\hline 9 & 1.144 \\
\hline 10 & 1.215 \\
\hline 11 & 1.279 \\
\hline 12 & 1.335 \\
\hline 13 & 1.386 \\
\hline 14 & 1.430 \\
\hline 15 & 1.468 \\
\hline 16 & 1.502 \\
\hline 17 & 1.530 \\
\hline 18 & 1.555 \\
\hline 19 & 1.574 \\
\hline 20 & 1.590 \\
\hline 21 & 1.602 \\
\hline 22 & 1.611 \\
\hline 23 & 1.617 \\
\hline 24 & 1.619 \\
\hline 25 & 1.619 \\
\hline 26 & 1.617 \\
\hline 27 & 1.612 \\
\hline 28 & 1.605 \\
\hline 29 & 1.596 \\
\hline 30 & 1.586 \\
\hline 31 & 1.574 \\
\hline 32 & 1.560 \\
\hline 33 & 1.545 \\
\hline 34 & 1.529 \\
\hline 35 & 1.511 \\
\hline 36 & 1.493 \\
\hline 37 & 1.474 \\
\hline 38 & 1.454 \\
\hline 39 & 1.433 \\
\hline
\end{tabular}


Table 4 Age weights for discrete time (Continued)

\begin{tabular}{|c|c|}
\hline$\overline{40}$ & 1.412 \\
\hline 41 & 1.390 \\
\hline 42 & 1.367 \\
\hline 43 & 1.345 \\
\hline 44 & 1.322 \\
\hline 45 & 1.298 \\
\hline 46 & 1.275 \\
\hline 47 & 1.251 \\
\hline 48 & 1.227 \\
\hline 49 & 1.204 \\
\hline 50 & 1.180 \\
\hline 51 & 1.156 \\
\hline 52 & 1.132 \\
\hline 53 & 1.109 \\
\hline 54 & 1.085 \\
\hline 55 & 1.062 \\
\hline 56 & 1.038 \\
\hline 57 & 1.015 \\
\hline 58 & 0.992 \\
\hline 59 & 0.970 \\
\hline 60 & 0.947 \\
\hline 61 & 0.925 \\
\hline 62 & 0.904 \\
\hline 63 & 0.882 \\
\hline 64 & 0.861 \\
\hline 65 & 0.840 \\
\hline 66 & 0.819 \\
\hline 67 & 0.799 \\
\hline 68 & 0.779 \\
\hline 69 & 0.759 \\
\hline 70 & 0.740 \\
\hline 71 & 0.721 \\
\hline 72 & 0.703 \\
\hline 73 & 0.684 \\
\hline 74 & 0.666 \\
\hline 75 & 0.649 \\
\hline 76 & 0.632 \\
\hline 77 & 0.615 \\
\hline 78 & 0.598 \\
\hline 79 & 0.582 \\
\hline 80 & 0.566 \\
\hline 81 & 0.551 \\
\hline 82 & 0.536 \\
\hline 83 & 0.521 \\
\hline 84 & 0.507 \\
\hline
\end{tabular}

Table 4 Age weights for discrete time (Continued)

\begin{tabular}{ll}
\hline 85 & 0.493 \\
86 & 0.479 \\
87 & 0.465 \\
88 & 0.452 \\
89 & 0.439 \\
90 & 0.427 \\
91 & 0.415 \\
92 & 0.403 \\
93 & 0.391 \\
94 & 0.380 \\
95 & 0.369 \\
96 & 0.358 \\
97 & 0.348 \\
98 & 0.337 \\
99 & 0.327 \\
\hline
\end{tabular}

\section{Appendix}

What about age weighting in discrete time?

The basic age-weighting function used in FRH and elsewhere is:

$$
a(x)=C x e^{-\beta x}=0.1658 x e^{-0.04 x}
$$

In general, this same age weighting function can be used in discrete time. Equation (5) implies, however, an age weight of 0 during the first year of life (birth through $1^{\text {st }}$ birthday), even though in continuous time $\mathrm{a}(\mathrm{x})$ grows from 0 at birth to 0.159 at one year.

To allow for the first year of life to have a positive age weight, the basic age weighting function can be adjusted to the mid-point for each year, so that:

$$
\begin{aligned}
a(x) & =C(x+0.5) e^{-\beta(x+0.5)} \\
& =0.1658(x+0.5) e^{-0.04(x+0.5)}
\end{aligned}
$$

This formulation allows the first year of life in discrete time to have a positive age weight (0.08126), and this age weight for the $1^{\text {st }}$ year of life is identical (to 5 decimal places) to the integral of (5) evaluated over 0 to 1 .

For the future, the logic of using 0.1658 in the ageweighting function for cost-effectiveness analyses, is not especially solid. With $\beta=0.04, C=0.1658$ was chosen so that global DALYs were the same with and without age weighting in the 1990 global burden of disease study [16]. With the new 2010 GBD estimates, the future of $\mathrm{C}=0.1658$ seems limited.

As an alternate, the approach suggested in the 2006 GDB report for values of $\beta$ other than 0.04 can be followed. In short, the value of $C$ can be chosen so that 
total years added up to 100 when age weighted or not [16]. Using this same logic in discrete time, Table 4 shows a spreadsheet organized to identify $C$ with $\beta=$ 0.04 . Once the age weight is included in the spreadsheet for any value of $C$, the value of $C$ can be adjusted until the sum of the column over 100 years adds up to $100(\mathrm{C}=0.176116)$. The age weights from Table 4 can be used to add age weighting into DALY calculations (if age weighting is included in the analysis).

\section{Abbreviation}

DALY: Disability adjusted life year lost.

\section{Competing interests}

The author declares that he have no competing interest.

\section{Acknowledgements}

This paper was developed as part of my teaching responsibilities in the Department of International Health at Boston University, which provides funding for these teaching activities. I would like to thank my students at Boston University for their patience, as the approach outlined in this paper was refined. Many thanks also to Sydney Rosen for her advice and input into the manuscript.

Received: 24 June 2013 Accepted: 29 July 2013

Published: 8 August 2013

\section{Reference}

1. World Bank: World Development Report, Investing in Health 1993. New York, New York: Published for the World Bank, Oxford University Press; 1993.

2. Murray CJ: Quantifying the burden of disease: the technical basis for disability-adjusted life years. Bull World Health Organ 1994, 72(3):429-45.

3. Murray CJ, Acharya AK: Understanding DALYs (disability-adjusted life years). J Health Econ 1997, 16(6):703-30

4. Fox-Rushby J, Hanson K: Calculating and presenting disability adjusted life years (DALYs) in cost-effectiveness analysis. Health Policy Plan 2001, 16(3):326-331

5. Phanthunane $P$, et al: Cost-effectiveness of pharmacological and psychosocial interventions for schizophrenia. Cost Effectiveness and Resource Allocation 2011, 9(1):6.

6. Amarasinghe A: Cost-effectiveness implications of GP intervention to promote physical activity: evidence from Perth. Australia. Cost Effectiveness and Resource Allocation 2010, 8(1):10.

7. Rubinstein A, et al: Generalized cost-effectiveness analysis of a package of interventions to reduce cardiovascular disease in Buenos Aires. Argentina. Cost Effectiveness and Resource Allocation 2009, 7(1):10.

8. Bachmann M: Cost effectiveness of community-based therapeutic care for children with severe acute malnutrition in Zambia: decision tree model. Cost Effectiveness and Resource Allocation 2009, 7(1):2.

9. Hansen K, Chapman G: Setting priorities for the health care sector in Zimbabwe using cost-effectiveness analysis and estimates of the burden of disease. Cost Effectiveness and Resource Allocation 2008, 6(1):14.

10. Dalziel K, Segal L, Mortimer D: Review of Australian health economic evaluation - 245 interventions: what can we say about cost effectiveness? Cost Effectiveness and Resource Allocation 2008, 6(1):9.

11. Robberstad B, Hemed Y, Norheim O: Cost-effectiveness of medical interventions to prevent cardiovascular disease in a sub-Saharan African country - the case of Tanzania. Cost Effectiveness and Resource Allocation 2007, 5(1):3.

12. Bishai $D$, Hyder $A$ : Modeling the cost effectiveness of injury interventions in lower and middle income countries: opportunities and challenges. Cost Effectiveness and Resource Allocation 2006, 4(1):2.

13. Shim E, et al: Evaluating the cost-effectiveness of rabies post-exposure prophylaxis: A case study in Tanzania. Vaccine 2009, 27(51):7167-7172.
14. Elbasha EH: Discrete time representation of the formula for calculating DALYs. Health Econ 2000, 9(4):353-365.

15. Drummond MF: Methods for the Economic Evaluation of Health Care Programmes. 3rd edition. Oxford: Oxford University Press; 2005.

16. Mathers CD, et al: Chapter 5: Sensitivity and Uncertainty Analyses for Burden of Disease and Risk Factor Estimates. In Global Burden of Disease and Risk Factors. Edited by Lopez AD. Washington, D.C: The World Bank; 2006.

doi:10.1186/1478-7547-11-18

Cite this article as: Larson: Calculating disability-adjusted-life-years lost (DALYs) in discrete-time. Cost Effectiveness and Resource Allocation 2013 11:18

\section{Submit your next manuscript to BioMed Central and take full advantage of:}

- Convenient online submission

- Thorough peer review

- No space constraints or color figure charges

- Immediate publication on acceptance

- Inclusion in PubMed, CAS, Scopus and Google Scholar

- Research which is freely available for redistribution 